\title{
Online-Sprachunterricht mit der PDL (Sprachpsychodramaturgie): Wege zu einer virtuellen Begegnung
}

\section{Erfahrungen mit Psychodrama-Online in der Fremdsprachenpädagogik}

\section{Monica Baudracco-Kastner}

Angenommen: 21. Januar 2022 / Online publiziert: 16. Februar 2022

(C) Springer Fachmedien Wiesbaden GmbH, ein Teil von Springer Nature 2022

Zusammenfassung Dieser Artikel der Zeitschrift für Psychodrama und Soziometrie befasst sich mit der digitalen Umsetzung psychodramatischer Arbeit im pädagogischen Anwendungsbereich des Sprachunterrichts mit der PDL (Sprachpsychodramaturgie). Im Fokus steht die Reflexion über die Möglichkeit die gruppenorientierten Erwerbs- und Lernprozesse, die dem PDL-Ansatz zugrunde liegen, online wirkungsvoll zu gestalten und Begegnungen im psychodramatischen Sinne zu ermöglichen. Die Chancen und Risiken der neuen, virtuellen Arbeitsumgebung für die Lernerfahrung im Sprachunterricht werden anhand von praktischen Beispielen und Feedbacks näher beleuchtet. Digitale Formate werden im Rahmen einer gesellschaftlichen Entwicklung betrachtet, die virtuelle Medien nicht mehr als Alternative für Technikbegeisterte bereithält, sondern zur Notwendigkeit für viele Bereiche des beruflichen und privaten Lebens vieler Menschen versteht.

Schlüsselwörter Digitalisierung · Erwachsenenbildung · Online · PDL · Psychodrama - Sprachpsychodramaturgie - Sprachunterricht - Unterrichtsformate · Digitale Lehre

Monica Baudracco-Kastner $(\triangle)$

Alter Stadtgraben 13, 91541 Rothenburg o.d.T., Deutschland

E-Mail: mail@ mbklearning.net 


\title{
Online- language lessons with the PDL (Psychodramaturgy For Language Acquisition): Ways to a virtual encounter
}

Online-Psychodrama practice for Foreign Language Education

\begin{abstract}
This article deals with the digital implementation of psychodrama in the pedagogical area of language teaching with the PDL Method (Psycho-dramaturgy for Language Acquisition). It reflects on the possibilities of effectively shaping the group-oriented learning processes that underlie the PDL approach in the virtual environment and of enabling encounters in the psycho-dramatic sense. Practical examples highlight the opportunities and risks of a new, virtual environment as it relates to the language learning experience. Digital formats are viewed within the context of a social development that no longer holds virtual media as the prerogative of technology enthusiasts, but rather as a necessity in many areas of our professional and private lives.
\end{abstract}

Keywords Digitalization · Adults Education · Language Learning · Learning Formats · Online · PDL · Psychodramaturgy for Language Acquisition · Psychodrama $\cdot$ Digital Education

\section{Einleitung}

Seit Anfang der Coronavirus-Pandemie im Frühjahr 2020 ist eine Beschleunigung von Digitalisierungsprozessen und eine Verbreitung virtueller Kommunikationskanäle in allen Bereichen des öffentlichen und privaten Lebens zu beobachten. Während es am Anfang noch darum ging, eine kurzfristige Alternative anzubieten, entwickelte sich dann immer mehr das Bedürfnis, die Angebote im digitalen Medium als eigenständiges Format mit eigener Qualität zu gestalten und zu etablieren. Mittlerweile ist es klar, dass Online-Veranstaltungen ein Teil des gesellschaftlichen Miteinanders geworden sind und bleiben werden. Nicht mehr alternativ, sondern ergänzend zu bestehenden Formaten. Neben der Reduktion, die wir im Virtuellen erleben, werden uns auch die spezifischen Merkmale und die Vorteile des Digitalen bewusst, auf die wir nicht mehr verzichten wollen.

In diesem Artikel befasse ich mich mit der psychodramatischen Arbeit im pädagogischen Bereich des Fremdsprachenunterrichts. Ich werde Beispiele der Umsetzung von Online-Sprachkursen mit der PDL Methode (Sprachpsychodramaturgie) aufzeigen und anhand meiner Erfahrungen diese besondere Form des digitalen Lernens anhand von Chancen und Risiken näher beleuchten.

\section{Prinzipien und Techniken der Sprachpsychodramaturgie (PDL)}

Online-Sprachunterricht ist bisher vorwiegend als asynchrone Lösung für die individuelle Arbeit vorhanden, als zusätzliches Angebot von Sprachschulen und Institutionen oder als digitale Applikation. Diese Sprachkurse bieten für unterschiedliche Niveaustufen meist Audio- oder Videomaterial zu verschiedenen Themen und 
Übungen wie z.B. Lückentexte, die online durchgeführt und ausgewertet werden. Auch an Hochschulen wird die digitale Lehre weiterentwickelt und als Ergänzung zu Präsenzveranstaltungen angeboten. Die Interaktion begrenzt sich in den meisten E-Learning oder Blended Learning Formaten auf Foren und Plattformen, in denen sich die Lernenden austauschen, mit den Lehrenden kommunizieren oder erledigte Aufgaben hochladen. Live-Sprachunterricht im digitalen Medium ist selten zu finden und die wenigen Angebote nutzen vorwiegend eine videogestützte Vortragsfunktion. Im besten Fall wird die Chatfunktion noch als Interaktionsmöglichkeit genutzt.

Diese Art von Sprachunterricht basiert auf der traditionellen Konzeption von Spracherwerb, z. B. durch das Auswendiglernen von Wortschatz und Grammatikregeln und das Erlernen von vorgefertigten sprachlichen Strukturen für bestimmte kommunikative Situationen. Diese Konzeption hat (unabhängig von der Art der Umsetzung, in Präsenz oder digital) wenig gemeinsam mit dem auf der PDL Methode basierenden Sprachunterricht, der Gegenstand dieses Artikels ist. Die Grundzüge der Sprachpsychodramaturgie und das darunterliegende Menschenbild haben viel mit den Konzepten von Begegnung, Handeln und schöpferischer Spontanität im Sinne Morenos gemein. Sie sind sowohl Hintergrund wie auch Ausgangspunkt meiner Überlegungen für eine digitale Umsetzung des psychodramatischen Sprachunterrichts. Daher möchte ich sie kurz skizzieren, bevor ich im Weiteren konkreter auf die Gestaltungsmöglichkeiten des Online-Unterrichts eingehen werde.

Die PDL (psychodramaturgie linguistique, übersetzt Sprachpsychodramaturgie) ist ein teilnehmer- und gruppenorientierter Ansatz für den Fremdsprachenerwerb, der 1977 von Bernard Dufeu und seiner Frau Marie initiiert wurde. Dieser wird seitdem kontinuierlich weiterentwickelt und angepasst. Die PDL stellt den Menschen mit seinem Ausdruckswunsch in den Mittelpunkt des Unterrichts. Dies steht im Gegensatz zu einem lehrwerkszentrieten Unterricht, bei dem die Inhalte im Fokus stehen. Dufeu beschreibt seinen Ansatz als eine „Pädagogik des Seins“, in der die Sprache vom Anfang an Mittel zur Kommunikation und Begegnung ist, gegenüber einer „Pädagogik des Habens“, bei der die sprachlichen Strukturen Gegenstand des Lernprozesses sind und die LehrerInnen als reine WissensvermittlerInnen fungieren (Dufeu 2003, S. 25-74).

In der Sprachpsychodramaturgie sind die Inhalte nicht im Voraus festgelegt, sondern entstehen durch die Teilnehmenden, die sich in Rahmenaktivitäten begegnen. Die Sprache folgt dem Ausdruckswunsch der Beteiligten und wird unter anderem durch psychodramatische Techniken wie das Doppeln oder das Spiegeln von der Leitung ,geliefert“(Dufeu 2013, S. 175-176) . Die Grundhypothese ist dabei, dass die Herstellung eines direkten Bezugs zwischen dem oder der SprecherIn und seinen/ihren Aussagen den Erwerb der Fremdsprache erleichtert (Dufeu 2003, S. 388). Auch in der PDL verläuft der Lernprozess in drei Phasen: Erwärmung ${ }^{1}$ (eine Übung, die Spontaneität weckt und auf die folgende Aktivität vorbereitet), Aktion (eine Aktivität, in der die Sprache in Interaktion erlebt und erfahren wird), Integration (die sprachliche Reflexion, die dazu beiträgt, die aktuellen Strukturen und den Wortschatz zu erweitern und zu festigen). Auch das Spiel und die Rollenübernahme haben einen wichtigen Platz und auch hier wurden Verfahren des psychodramatischen Rollen-

\footnotetext{
${ }^{1}$ Dufeu verwendet hier eher den Begriff Aufwärmung
} 
spiels übernommen. Die Wirkungsweise von Übungen und Techniken ist ähnlich wie im Psychodrama und geht weit über den Spracherwerb hinaus, da sie den Menschen in seiner Ganzheitlichkeit anspricht und gleichzeitig allgemeine kommunikative und soziale Kompetenzen trainiert. Die Abgrenzung zum Psychodrama ist eher bei der Zielsetzung zu finden. Das Ziel im Kontext des Sprachunterrichts ist in erster Linie die Beherrschung kommunikativer Situationen in der Fremdsprache. Die tiefere biographische Dimension des Einzelnen und die Dynamik der Gruppe wirken zwar mit, sind aber nicht Gegenstand des Lernprozesses. Um den Teilnehmenden einen gewissen Schutz zu bieten und mögliche Hemmungen abzubauen, nutzt die PDL die Ebene der Imagination. Die Rollen die miteinander interagieren sind meist fiktive Personen oder Gegenstände. Die Ausgestaltung der Rolle sagt zwar auch etwas über die beteiligte Person aus, aber die spezifische Bedeutung wird im Vergleich zum Psychodrama nicht tiefergehend exploriert.

PDL TrainerInnen nutzen Spontaneität und Kreativität, um mit den Teilnehmenden Welten zu erschaffen, in denen die Lust am Ausdruck entsteht und begleitet wird (dazu s. auch: Montali 2013, S. 14-20). Dazu braucht es eine vertraute Atmosphäre, die gleichzeitig die kognitive und emotionale Dimension anspricht und die es den Einzelnen ermöglicht, in ihrem eigenen Rhythmus und gleichzeitig von den anderen zu lernen. Es geht also zuallererst darum, einen sicheren und geschützten Rahmen anzubieten, damit sich alle trauen, sich in der Fremdsprache auszuprobieren. Im Folgenden werde ich beschreiben, wie Sprachunterricht auch in der virtuellen Umgebung so gestaltet werden kann, dass dies gelingt.

\section{Arbeitsphasen der Sprachpsychodramaturgie und Möglichkeiten der digitalen Umsetzung}

\subsection{Virtuellen Rahmen gestalten}

Eine Fremdsprache sprechen heißt, in eine fremde Welt einzutreten und die vertraute Welt der eigenen Muttersprache zu verlassen. Eine Fremdsprache zu erlernen heißt für die Lernenden, die Stufen der kindlichen Entwicklung „neu“ zu durchlaufen. Hiervon leitet die PDL ihre Techniken und ihre Progression $\mathrm{ab}^{2}$. Von der Wahrnehmung des eigenen Selbst über die progressive Differenzierung zu anderen bis hin zur sozialen Interaktion ${ }^{3}$. Diese Betrachtung des Spracherwerbs erklärt die Hemmungen und Widerstände, die beim Erlernen einer Fremdsprache zu Tage treten können und oft im traditionellen (Schul-) Unterricht eine Hürde zum Lernfortschritt darstellen.

In digitalen Formaten bekommt die Gestaltung eines sicheren Rahmens (NéveHanquet, Crespel 2020, S. 27-29) schon wegen des Mediums an sich eine besondere Bedeutung. Die virtuelle Präsenz stellt eine Verfremdung dar, die im Falle des Sprachunterrichts zu der Verfremdung durch die neue Sprache hinzukommt. Unsi-

\footnotetext{
2 Dufeu spricht bei der PDL von einer relationellen Progression, im Gegensatz zu einer im Voraus festgelegten sprachlichen oder grammatikalischen Progression, auf die der traditionelle, lehrwerkzentrierte Sprachunterricht basiert

${ }^{3}$ Zur Entwicklung der Rollenebenen in Morenos Theorie s. Schacht (2003).
} 
cherheiten im Umgang mit der Technik können zu Frustration und unangenehmen Gefühlen führen (,,ich komme nicht mit“, ,,ich bin langsamer als die anderen“) und den Kontakt erschweren. Außerdem ist die Technik ein weiteres Element, das einerseits viele Möglichkeiten bietet, andererseits jederzeit auch unerwartete Schwierigkeiten bereiten kann, wie zum Beispiel Verbindungsprobleme, die den Kursverlauf stören und bei den Betroffenen großen Druck auslösen können.

Ich sehe diese Phase, die ich Rahmen gestalten nenne, vor der Erwärmung als eigenständigen Abschnitt einer Online-Veranstaltung, der durchdacht und in den Ablauf integriert gehört (Baudracco-Kastner, 2020, S. 8-15). Die Leitung hat die Aufgabe, einen Raum zu eröffnen und die Teilnehmenden abzuholen und einzuladen, es sich in diesem Raum bequem zu machen. Es soll ein WIR-Gefühl entstehen, dass die Gruppe erst arbeitsfähig macht. In einer Präsenzveranstaltung tragen die Aufteilung und Ausstattung der Räume, sowie die Abläufe und Rituale eines Treffens dazu bei, Orientierung zu geben und eine Atmosphäre des Vertrauens herzustellen. Das hilft den Teilnehmenden ihren Platz in der Gruppe zu finden. Doch wie geschieht das über ein digitales Medium? Wie kommen wir miteinander in Beziehung, wenn wir uns physisch nicht im gleichen Raum befinden? Welche Regeln bestimmen das virtuelle Zusammensein und sind diese allen Beteiligten bekannt?

Zur Phase Rahmen gestalten gehört auch das Ankommen im virtuellen Gruppenraum. In der Regel wird der Raum mindestens 15 min vor dem offiziellen Start eröffnet, sodass die Teilnehmenden sich schon einwählen und die Technik testen können. Im Sprachunterricht nutze ich diese Phase auch, um einen ersten Kontakt mit der Fremdsprache anzubieten: Während meine Kamera meist noch ausgeschaltet ist, lasse ich Musik laufen, deren Wahl mit der Fremdsprache und mit Themen und Inhalten zu tun hat, die im jeweiligen Kurs dran sind oder waren.

Nach dem Betreten des virtuellen Raums können die Teilnehmenden noch bei sich bleiben und Musik hören oder mit anderen Anwesenden in Kontakt treten. Ich begrüße die Teilnehmenden mit kurzen Sätzen in der Fremdsprache im Chat um meine Präsenz zu signalisieren. Die erhaltenen Feedbacks bestätigen immer wieder, dass die bewusste Gestaltung dieser inoffiziellen Phase den Teilnehmenden hilft, sich abgeholt zu fühlen und anzukommen.

\subsection{Erwärmung: Virtuell in Beziehung kommen}

Ziel der Erwärmungsphase ist es, in der PDL wie auch im Psychodrama, die Gruppe zu lockern und zu vernetzen, alle Sinne zu wecken und miteinzubeziehen, um Spontanität und Kreativität zu fördern. Im Sprachunterricht kommen bei der Erwärmung noch zusätzliche Aufgaben hinzu: Zum Beispiel die Teilnehmenden mit der Fremdsprache vertraut zu machen, vorhandenes Wissen zu aktivieren und eine Atmosphäre zu fördern, in der eventuelle sprachliche Blockaden aufgelöst werden. Durch besondere Erwärmungsmethoden gelingt es, die Gruppe schnell arbeitsfähig zu machen (Buckel, Reineck, Anderl 2021, S. 55). In der PDL werden die Teilnehmenden immer mit einer Aufwärmung auf die folgende Hauptübung vorbereitet. Die Aufwärmung ist auf die geplante Aktivität abgestimmt und fördert die Haltungen und Fähigkeiten, die in der darauffolgenden Phase gebraucht werden. 
Um die aktive Teilnahme so niedrigschwellig wie möglich zu halten und die Teilnehmenden mit sich selbst, mit den anderen und mit dem Medium in Kontakt zu bringen, nutze ich die Galerieansicht ${ }^{4}$ für spezifische Aktivitäten, die auch die körperliche Dimension und das jeweilige Umfeld der Teilnehmenden miteinbeziehen. Der klassische Raumlauf am Anfang einer psychodramatischen Sitzung kann auch bei einer Online-Veranstaltung übernommen werden, unter Berücksichtigung der räumlichen Bedingungen der Teilnehmenden. So werden diese zunächst eingeladen aufzustehen und sich in einer Reichweite zu bewegen, in der sie noch die Stimme der Leitung hören können, die sie anleitet. Man kann dann mit den auditiven und visuellen Reizen in den jeweiligen Räumen der Teilnehmenden arbeiten und ihre Aufmerksamkeit auf die Geräusche lenken, die sie umgeben oder sie auffordern, ein Fenster, eine Tür oder einen Schrank aufzumachen und sich ein Bild einzuprägen oder einen Gegenstand mitzunehmen. Da es in dieser Phase auch um eine sprachliche Aufwärmung geht, werden die Teilnehmenden eingeladen, Laute oder Worte in der Fremdsprache kommen zu lassen, die sich spontan ergeben und damit zu experimentieren (z. B. ein Wort mit unterschiedlichen Betonungen auszusprechen).

Nach dieser kurzen Bewegungsphase kommen die Teilnehmenden an ihren Arbeitsplatz zurück und teilen, je nach Anleitung, etwas mit der Gruppe. Es kann z. B. eine Haltung sein, die ihr Befinden widerspiegelt, begleitet von einem Wort in der Fremdsprache. Die Gruppe übernimmt die Haltung und das Wort als Echo und Spiegel. Oder es kann ein Gegenstand sein, den die Teilnehmenden in die Kamera halten und der sich im Rollentausch vorstellt bzw. als Symbol für das jeweilige Befinden gilt (z.B.: „Ich fühle mich frisch wie eine blühende Zimmerpflanze“). Je nach Kenntnisstand der Gruppe erfordert die Aufgabe mehr oder weniger komplexe sprachliche Äußerungen. Durch das Teilen von Eindrücken und Einzelheiten aus der individuellen Umgebung stellt sich eine Beziehung unter den Teilnehmenden ein, es entstehen Neugier für die anderen und Gesprächsstoff für die folgenden Aktivitäten.

Um die Gruppenkohäsion zu fördern und auf die folgende Aktivität vorzubereiten, folgt meist eine Gruppenübung, in der sich die Teilnehmenden in der Fremdsprache äußern oder in Bezug auf bestimmte Kriterien und Fragen positionieren und schrittweise in eine Interaktion miteinander kommen. Je nach Niveaustufe werden überwiegend paraverbale Elemente (Gestik, Mimik) oder komplexere sprachliche Äußerungen eingesetzt. An dieser Stelle eignen sich aktionssoziometrische Übungen sehr gut. Diese lassen sich auch online unkompliziert umsetzen und sie können je nach Schwerpunkt und Thema beliebig angepasst und variiert werden. Der virtuelle soziometrische Step-in Zirkel mit Abdecken der Kamera mit einem bunten Haftnotizzettel und Aufdecken der Kamera, um eine Frage zu beantworten, hat sich mittlerweile in sämtlichen Formaten der Online-Arbeit etabliert. Weit über die psychodramatischen Settings hinaus. Auch das Positionieren nach einem vorgegebenen Kriterium lässt sich mit dem Zeichnen einer Linie auf dem virtuellen Whiteboard, auf der die Teilnehmenden einen Stempel setzen oder ihren Namen schreiben, leicht umsetzen. Sprachlich ist diese letzte Übung auf doppelter Weise effektiv: Schon bei den Anweisungen bezüglich der eigenen Position auf der Linie werden Strukturen

\footnotetext{
${ }^{4}$ Die Plattform Zoom nennt Galerie die Ansicht, in der alle Teilnehmende in einer Kachel gleicher Größe mit ihrem Kamerabild sichtbar sind.
} 
in der Fremdsprache geübt. Nachdem alle ihre Position gefunden haben, können dann die Teilnehmenden noch etwas dazu sagen und nutzen somit weitere Wörter und sprachliche Mittel.

Die Aufmerksamkeit ist hier auf die angesprochenen Themen und die Visualisierung gelenkt und nicht auf die Sprachproduktion. Dies führt dazu, dass ein authentischer Ausdruckswunsch entsteht, der sich in der Fremdsprache äußert.

\subsection{Hauptaktivität: Aktive Anwendung der Fremdsprache}

„Wer erzählen will, muss sich eine Welt erschaffen. Die Worte kommen dann fast von selbst.“ Umberto Eco

Die Aktionsphase wird in der PDL je nach Kenntnisstand der Teilnehmenden unterschiedlich gestaltet und unterteilt sich in: Ausdruck, sprachliche Erweiterung und Wiederaufnahmetechniken. Während bei fortgeschrittenen Lernenden gruppendramaturgische Aktivitäten und Arrangements zum Einsatz kommen, die sich am Repertoire des Psychodramas bedienen, findet bei AnfängerInnen in den ersten Phasen eines Kurses eine individuelle Begegnung mit der Fremdsprache statt. Hier wird die Doppeltechnik eingesetzt, die zwar im psychodramatischen Doppeln ihren Ursprung hat, jedoch im Sprachunterricht überwiegend das Ziel verfolgt, erste Versuche des sprachlichen Ausdrucks in der Fremdsprache zu begleiten (Dufeu 2013, S. 6-10).

Nach dem Prinzip der PDL ,Wenn es ihre Worte sind, kann es ihre Sprache werden“ (Dufeu 2003, S. 39), entstehen die Themen des Sprachunterrichts bei allen Niveaustufen aus dem Ausdruckswunsch der Teilnehmenden. Die Art der Rahmenaktivitäten und der Auslöser des sprachlichen Ausdrucks werden von der Leitung so gewählt, dass vorgegebene bzw. passende sprachliche Strukturen im Laufe der Aktivität entstehen und diese eingeübt und anschließend reflektiert werden. Diese sind aber nie Gegenstand des Unterrichts, sondern Kommunikationsmittel in der Situation. Es soll also, mit den Worten von Umberto Eco, eine „Welt“ erschaffen werden, in der sich die Sprache ,wie von selbst“ entwickelt und mit Unterstützung der Leitung zum Erwerb neuer Fähigkeiten und Kenntnisse führt.

Im Online-Setting bieten sich, sowohl für das Doppeln als auch für die Gruppendramaturgie vielfältige Möglichkeiten, mit Bildern, Musik und Texten als Auslöser $\mathrm{zu}$ arbeiten. Mit einer Internetrecherche, im Vorfeld oder während der Aktivität, kann man auf passendes Material für die Aktivitäten zugreifen und dieses in den Prozess einbauen und mit den Teilnehmenden teilen.

Bei einem Italienischkurs im April 2020 wurde das Bild einer leeren Straße als Impuls angeboten. Das Bild wurde über die Funktion Bildschirm freigeben für alle sichtbar und bearbeitbar gemacht. Die Teilnehmenden wurden eingeladen, die leere Straße zu beleben und sie zum virtuellen Treffpunkt zu machen. Das Bild wurde mit Zeichnungen und Worten gestaltet und durch Bewegen des Cursors auf dem Bild wurde sichtbar, wer sich wo auf dem Bild befindet. Nach der Anwärmung der ganzen Gruppe fanden vor der Kulisse des Bildes Begegnungen statt und kleine Dialoge entstanden, die nach der Doppeltechnik begleitet und unterstützt wurden. 


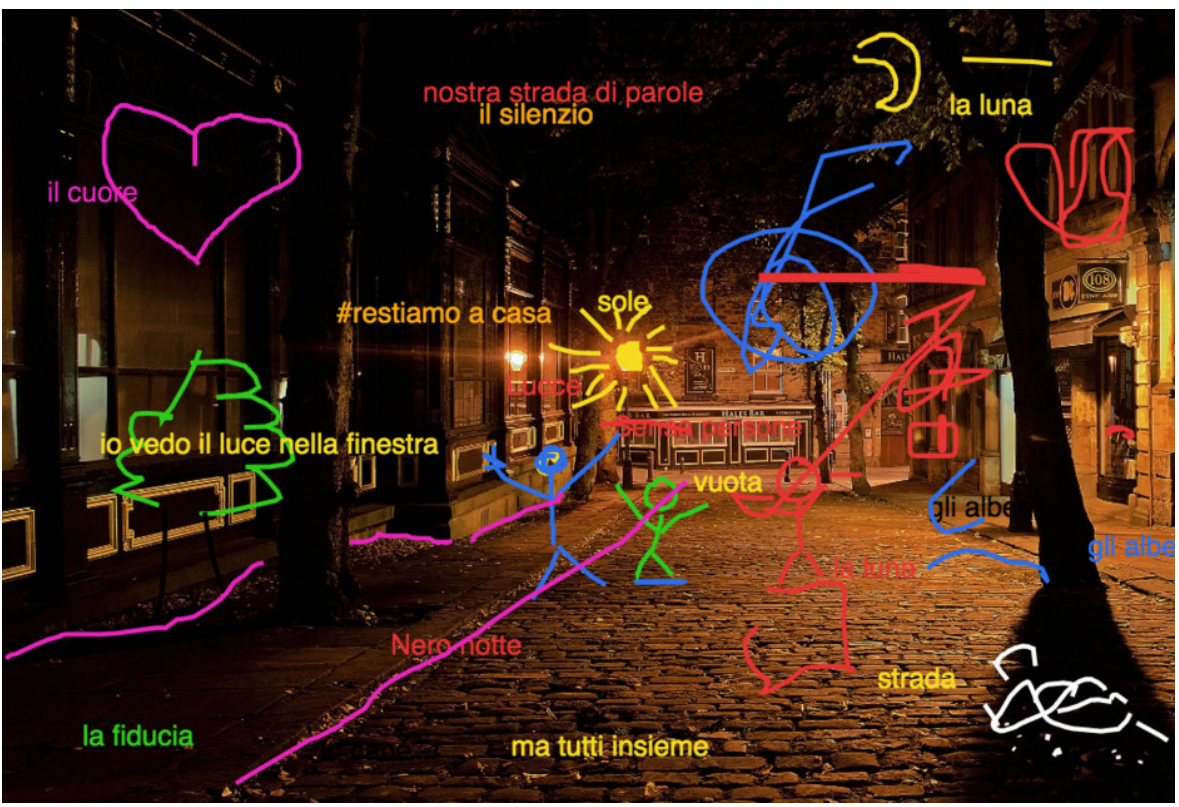

Abb. 1 Bühnenbild einer Aktivität im Italienischunterricht, entstanden bei einem VHS Kurs im April 2020. (Originalbild: pixabay (lizenzfrei))

Die entstandenen Bühnenbilder können nach der Aktivität gespeichert und der Gruppe zugänglich gemacht werden, was für eine sprachliche Reflexion im Nachgang hilfreich sein kann (Abb. 1).

Es ist auch möglich, verschiedene Bilder (z.B. Landschaften) zur Auswahl zu stellen und einzelne ProtagonistInnen durch das Doppeln auf verbalem Impuls ${ }^{5}$ beim Aufbau einer vom Bild inspirierten sprachlichen Sequenz zu unterstützen.

Im Online-Kontext fehlt bei diesen Aktivitäten der „,echte“ Kontakt und wichtige prosodische Merkmale werden nur eingeschränkt wahrgenommen. Dies erschwert zwar das Verständnis, aber ich bekam auch oft die Rückmeldung, dass das digitale Medium eine zusätzliche Schutzfunktion bietet. Dadurch, dass man nicht unmittelbar für alle sichtbar ist, sondern in der eigenen vertrauten Umgebung bleibt, können die Hemmungen in der Sprachproduktion leichter zu überwinden sein. Sehr wichtig ist die Qualität des Audios: Hier ist darauf zu achten, dass sowohl die Leitung als auch die Teilnehmenden hochwertige und gut funktionierende Mikrophone und Kopfhörer nutzen. Unter diesen Voraussetzungen gelingt die sprachliche Produktion mit Unterstützung der Leitung, ähnlich gut wie in Präsenz. Schwieriger gestaltet sich das Doppeln der ProtagonistInnen durch Gruppenmitglieder, da diese nicht wie im Präsenzkurs hinter den ProtagonistInnen sitzen und ihnen sprachliche Vorschläge ins Ohr flüstern können. Die Interventionen der Gruppenmitglieder können verwirrend und störend wirken. Besser durchführbar ist hier das „Abklatschen“ einer Rolle durch

\footnotetext{
5 S. Dufeu (2003).
} 
ein vereinbartes Zeichen und das kurzfristige Einspringen einer anderen Person anstelle des oder der ProtagonistIn.

Mit fortgeschrittenen TeilnehmerInnen können in der Aktionsphase bereits verstärkt Aktivitäten der Gruppendramaturgie angewandt und mit imaginären Rollen und Situationen gearbeitet werden. Der aktiven Phase der Sprachproduktion folgen eine Aufladung (Wiederaufnahme und Erweiterung der entstandenen sprachlichen Sequenzen), eine пеие Begegnung (Wiederholung der Sequenz mit der Möglichkeit, einzelne Parameter oder die Rollenbesetzungen zu verändern) und ggf. eine Fortsetzung als schriftliche Übung. Die Aktivität Die Kissen, eine spezielle Methodik der PDL (Dufeu 2003, S. 190-193), wurde für die digitale Durchführung speziell erprobt und angepasst. Es geht in der Übung darum, imaginäre Rollen durch Projektionstechniken zu erfinden und diese in wiederholten Begegnungen interagieren zu lassen, wobei die Leitung die Funktion hat, den sprachlichen Ausdruck zu unterstützen und zu erweitern, sowie die Entstehung der Handlungen zu begleiten und durch den Einwurf neuer Parameter im Fluss zu halten.

Die Arbeit in zwei (oder mehr) Untergruppen wird durch die Nutzung von digitalen Gruppenräumen (Break-out-rooms), die abwechselnd von der Leitung begleitet werden, ermöglicht. In der klassischen Version in Präsenz findet der 1. Schritt der Übung schon in zwei Untergruppen statt, die in ihrer Mitte ein leeres Kissen haben, welches die imaginäre Person darstellt. Durch Angaben der Gruppenmitglieder wird die Rolle definiert, die später in der Situation interagieren wird. Um den kreativen Prozess leichter anzustoßen, biete ich in der Online-Variante im Vorfeld Bilder oder Texte als Auslöser für die Erfindung der Rollen an. Es können auch die Bilder von zwei unterschiedlichen Stühlen sein, auf denen jeweils eine imaginäre Person sitzt. Das jeweilige Bild kann dann von jeder Untergruppe durch Teilen des Bildschirms visualisiert werden. Die Teilnehmenden ordnen sich durch soziometrische Wahl der Rolle zu, die sie am meisten anspricht und arbeiten anschließend im Break-outroom an der Entstehung dieser Rolle. Wie in der klassischen Aktivität geschieht dies durch Angaben der Gruppenmitglieder (in der Fremdsprache). Die einzige Regel ist, dass der erste Vorschlag gilt (wenn z. B. A sagt, die imaginäre Person sei ein Mann, kann B die Aussage nicht korrigieren oder dieser widersprechen). In einem nächsten Schritt bestimmt die Untergruppe, wer aus ihren Reihen die Rolle übernimmt und imaginär auf dem Stuhl oder dem Kissen Platz nimmt. Es findet dann ein Interview durch die eigene Gruppe statt. Während dieser Phase und wenn die Personen ausreichend definiert sind, schaltet sich die Leitung nacheinander in die zwei Gruppenräume zu und gibt die Anweisung, dass die imaginäre Person ein Vorhaben realisieren möchte, wofür sie Hilfe braucht. Die Leitung kann hier außerdem die Rolle sprachlich unterstützen und durch Wiederaufnahme von Sätzen eventuelle Fehler unauffällig korrigieren. Die Gruppe einigt sich auf das Vorhaben und bereitet sich so auf die Begegnung der imaginären Rollen vor.

Durch Beenden der Break-out-rooms kommen alle Teilnehmenden zurück in die Galerieansicht. In der Online-Variante wird auf das Interview der Rollen durch eine Person aus der anderen Gruppe verzichtet, um durch den virtuellen Wechsel der Räume nicht zu verwirren. Stattdessen stellt ein/eine SprecherIn aus jeder Untergruppe die imaginäre Person und das Vorhaben vor. Dann werden von der Gesamtgruppe ein Ort und eine Zeit für ein Treffen der beiden imaginären Gestalten definiert und die 
zwei Teilnehmenden, die die Rolle übernommen haben, bleiben mit ihrem Kamerabild sichtbar, während die anderen ihre Kamera abdecken (die offene Kamera hat sich im digitalen Kontext als Zeichen dafür etabliert, dass die jeweiligen Personen auf der Bühne sind).

Es findet ein Dialog zwischen den beiden ProtagonistInnen statt. Die Leitung und die anderen Gruppenmitglieder können unterstützen, indem sie sprachliche Vorschläge machen, die von der jeweiligen Rolle übernommen werden können. Die Arbeit kann dann erneut in Gruppenräumen weitergehen, wo abwechselnd mit Unterstützung der Leitung der sprachliche Ausdruck erweitert wird. Es findet dann eine erneute Begegnung statt, ggf. mit einem Wechsel der Rollenbesetzung oder eines Parameters (z. B. kann das erneute Treffen nach einem Jahr oder nach dem Eintreten eines unerwarteten Ereignisses stattfinden).

Die Aktivität kann nach der mündlichen Sprachproduktion beendet werden, in die sprachliche Reflexionsphase übergehen oder auch mit einer schriftlichen Übung fortgesetzt werden (zum Beispiel das Verfassen eines Zeitungartikels über die Begegnung der imaginären Gestalten). Durch webbasierte Kommunikationstools, mit denen Dokumente oder Medieninhalte sich am Monitor von mehreren Personen gleichzeitig betrachten und bearbeiten lassen, können die Teilnehmenden in Untergruppen gemeinsam an einem Text schreiben, die Ergebnisse speichern und diese dann mit der ganzen Gruppe teilen.

\subsection{Reflexions- und Integrationsphase}

Nach der sprachlichen Produktion in der Aktionsphase begibt sich die Gruppe anschließend auf eine Reflexionsebene. Anders als im Psychodrama, handelt es sich in der PDL um eine sprachliche kognitive Auswertung und eine Vertiefung der geübten und erworbenen sprachlichen Strukturen. Die Leitung fragt typischerweise (statt nach einem Rollenfeedback) nach Worten und Sätzen, die bei den Teilnehmenden noch nachklingen und wiederholt oder geschrieben werden möchten bzw. noch erklärungsbedürftig sind. Zur gemeinsamen Visualisierung wird am Bildschirm ein Whiteboard oder eine sonstige virtuelle Pinnwand geteilt, wo Wörter und Sätze von den Teilnehmenden selbst oder von der Leitung aufgeschrieben werden. Dies dient im Nachgang als Dokumentation und Grundlage für die selbständige Arbeit der Teilnehmenden. In dieser Phase werden auch spontan entstandene grammatikalische Fragen beantwortet und mit Beispielen hinterlegt.

In den letzten Minuten eines digitalen Treffens finden kleine Rituale statt, die auf die sprachliche Integration von neuen Wörtern und Strukturen abzielen. Das kann durch das Wiederholen von Sätzen aus den Aktivitäten durch die Leitung oder das Vortragen eines thematisch passenden Gedichts sein, während die Teilnehmenden sich entspannt zurücklehnen und sich von der Fremdsprache berieseln lassen. Anschließend können sie gefragt werden, welche Worte und Sätze sie von der aktuellen Sitzung mitnehmen möchten, z. B. durch die Metapher des Koffers, der mit Wörtern bepackt werden kann oder eines Brunnens, aus dem man Worte und Sätze schöpfen kann. 
Das digitale Medium bietet vielfältige Möglichkeiten, die Abschlussphase auch effektvoll zu gestalten, zum Beispiel durch das Visualisieren von Bildern oder Videosequenzen oder das Abspielen von Musik.

\section{Abschließende Bemerkungen zur digitalen Umsetzung von Sprachunterricht mit der PDL Methode}

Wie aus den oben beschriebenen Beispielen deutlich wird, können für den Sprachunterricht die Techniken und Methoden der Psychodramaturgie auch online umgesetzt werden. Es geht darum, die Wirkfaktoren des Verfahrens zu berücksichtigen und Anpassungen an das digitale Medium vorzunehmen. Gleichzeitig kann man die Vorteile der Technik dazu nutzen, den virtuellen Raum choreographisch schön und einladend zu gestalten, oder gewisse Wirkungen sogar zu verstärken. Neben einigen Grenzen bieten Online-Formate auch Vorteile, die sich positiv auf die Prozesse auswirken und Bedingungen vereinfachen, so dass mehr Menschen eine Teilnahme ermöglicht wird. Die Gruppenzusammensetzung wird vielfältiger und der Austausch lebendiger, Netzwerke werden erweitert und Kontakte geknüpft, die über den Sprachkurs hinaus bestehen und auch zum gemeinsamen Lernen und Üben genutzt werden.

Die Frage, ob sich die verschiedenen Formen psychodramatischer Online-Arbeit in Zukunft etablieren oder eher als Notlösung für verrückte Zeiten archiviert werden, ist schwer zu beantworten. Klar ist jedoch, dass Digitalisierung weiter unser Leben prägen wird. Allein aus diesem Grund muss sich auch Bildung (ob Schul- oder Weiterbildung) auf den digitalen Weg begeben, um mit der Zeit zu gehen und auch weiterhin Menschen aller Altersstufen zu erreichen. Die aus der Not entstandenen positiven Erfahrungen in der Nutzung von digitalen Medien sollen als Bereicherung und Ergänzung bestehender Unterrichtsformen mitgenommen und integriert werden. Der interaktive Online-Sprachunterricht mit der PDL Methode bietet sich einerseits als Ergänzung von Präsenzunterricht an, um den logistischen Aufwand zu reduzieren und auch über größere Entfernungen hinweg eine Kontinuität im Gruppenprozess zu bieten. Andererseits können auch klassische, asynchrone Online-Sprachkurse von interaktiven Online-Sitzungen zwischendurch profitieren, die durch das gemeinsame Üben der Fremdsprache auch persönliche Beziehungen in der Gruppe ermöglichen.

Mein Fazit nach den Erfahrungen mit dem Online-Medium ist, dass auch wenn der Spracherwerb nicht von einer echten Kommunikation und echter Begegnung absehen kann, die Möglichkeiten eines interaktiven Online-Unterrichts dennoch eine bereichernde Ergänzung bzw. ein valider Ersatz sein können. Vor allem in den Fällen, in denen eine Präsenzveranstaltung schwer oder gar nicht realisierbar ist.

Der virtuelle Raum ist für den psychodramatischen Sprachunterricht zugänglich gemacht worden. Besonders für diejenigen, die sich dort ausprobieren und Begegnung erleben wollen. Darin liegt die Chance. 


\section{Literatur}

\section{Verwendete Literatur}

Baudracco-Kastner, M. (2020). Psychodrama goes online. Möglichkeiten virtueller psychodramatischer Arbeit für Training und Beratung. München: GRIN. https://www.grin.com/document/987681

Buckel, C., Reineck, U., \& Anderl, M. (2021). Praxishandbuch Soziodrama. Theorie, Methoden, Anwendung. Weinheim: Beltz.

Dufeu, B. (2003). Wege zu einer Pädagogik des Seins. Mainz: Éditions Psychodramaturgie.

Dufeu, B. (2013). Das Doppeln in der Psychodramaturgie. Zeitschrift für Psychodrama und Soziometrie, 12, 173-187. https://doi.org/10.1007/s11620-013-0193-x.

Montali, S. (2013). Italiano con l'immaginario. Tre canovacci di simulation globale. Bozen: Unversity Press.

Nève-Hanquet, C., \& Crespel, A. (2020). Facilitating collective intelligence. A handbook for trainers, coaches, consultants and leaders. New York: Routledge.

Schacht, M. (2003). Spontaneität und Begegnung. Zur Persönlichkeitsentwicklung aus der Sicht des Psychodramas. München: inScenario.

\section{Weiterführende Literatur}

Haus, B. (2016). Psychodrama in der Schule. Innovative Lernprozesse für alle. Norderstedt: Books on Demand, GRIN. http://www.grin.com/de/e-book/346901/psychodrama-in-der-schule-innovativelernprozesse-fuer-alle

Nonnenmacher, P. (2021). Psychodrama und Digitalisierung. Beziehungsstatus: „Es ist kompliziert!“. Zeitschrift für Psychodrama und Soziometrie. https://doi.org/10.1007/s11620-021-00603-2.

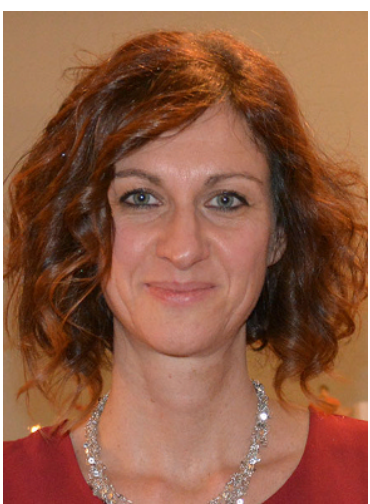

Monica Baudracco-Kastner Jg. 1973, M. A. in Germanistik und Anglistik (Dott.ssa in Lingue e Letterature Straniere Moderne), Psychodrama-Leiterin (DFP), ausgebildete PDL Trainerin und -Ausbilderin (Mitglied im internationalen PDL Verband). Personal- und Organisationsentwicklerin, freiberufliche Trainerin und Beraterin. Arbeitsschwerpunkte: Fremdsprachentrainings in der Erwachsenenbildung für Italienisch und DaF, LehrerInnenweiterbildung, Methodentrainings, Trainings für Online-Formate, Kommunikationstrainings, Organisationsberatung und Führungskräfteentwicklung. Lehrbeauftragte am Moreno Institut Stuttgart, an der IHK-Akademie Mittelfranken und an der Hochschule Ansbach. 